\title{
Intervenção educacional para promover o cuidado centrado na família em oncologia pediátrica: um estudo piloto
}

\author{
Educational intervention to promote Patient- and Family-Centered Care in pediatric oncology: a pilot study
}

Intervención educativa para promover la atención centrada en la familia en oncología pediátrica: un estudio piloto

Fernanda Ribeiro Baptista Marques ${ }^{1}$ (D) htps://orcid.org/0000-0003-1024-6787

Myriam Aparecida Mandetta ${ }^{2}$ (D) https://orcid.org/0000-003-4399-2479

\section{Resumo}

Objetivo: Avaliar o impacto de uma intervenção educacional no conhecimento, na percepção e na atitude dos profissionais da equipe de enfermagem em relação ao Modelo do Cuidado Centrado na Família.

Métodos: Quase experimento, com delineamento de amostras temporais equivalentes em três fases. Aplicou-se questionário da avaliação do conhecimento teórico, instrumento Percepção do Cuidado Centrado na Família equipe; Escala da Importância das Famílias nos Cuidados de Enfermagem - atitudes dos enfermeiros e entrevista semiestruturada na última fase. A análise descritiva e inferencial $(p \leq 0,05)$ foi aplicada para os dados quantitativos, e a análise qualitativa de conteúdo, para dados qualitativos.

Resultados: Os profissionais adquiriram conhecimentos sobre a importância de fornecer informação à família. Os profissionais aumentaram a atribuição de importância da inclusão da família nos cuidados.

Conclusão: A intervenção favoreceu a aquisição de novos conhecimentos teóricos, modificou conceito e atitudes com a família, possibilitando refletir sobre as barreiras institucionais e pessoais.

\section{Abstract}

Objective: To evaluate an educational intervention on the knowledge, perceptions, and attitudes of nursing team professionals regarding patient- and family-centered care.

Methods: Quasi-experimental, for equivalente time-samples design, in three phases. Were applied a theoretical knowledge questionnaire; the measurement instruments Perceptions of Family Centred Care - Staff (PFCC-S) Brazilian version and the scale; Families Importance in Nursing Care - Nurses Attitudes. Descriptive and inferential data analysis were conducted for the quantitative study $(p \leq 0,05)$. The Content Qualitative Analysis were conducted for qualitative data analysis.

Results: These professionals showed improvement in the acquisition of knowledge regarding the transmission of information to the family and increased the importance of including the family in care.

Conclusion: The education intervention that favored the assimilation of new knowledge, to change concepts and care attitudes to family. However, they realized that there were many barriers to collaborative practice with family.

\section{Resumen}

Objetivo: Evaluar el impacto de una intervención educativa en el conocimiento, percepción y actitud de los profesionales del equipo de enfermería en relación al Modelo de Atención Centrado en la Familia.

Métodos: Cuasiexperimental, con diseño de muestras temporales equivalentes en tres fases. Se aplicó cuestionario de evaluación de conocimientos teóricos, instrumento Percepción del Cuidado Centrado en la Familia - equipo; Escala de Importancia de la Familia en la Atención de Enfermería - Actitudes de enfermeras y entrevista semiestructurada en última fase. Se aplicó análisis descriptivo e inferencial $(p \leq 0.05)$ para datos cuantitativos y análisis de contenido cualitativo para datos cualitativos.

Resultados: Los profesionales adquirieron conocimientos sobre la importancia de brindar información a la familia. Los profesionales aumentaron la importancia de incluir a la familia en el cuidado.

Conclusión: La intervención favoreció la adquisición de nuevos conocimientos teóricos, modificó el concepto y las actitudes hacia la familia, permitiendo reflexionar sobre las barreras institucionales y personales.

\section{Descritores}

Enfermagem familiar; Relações profissional-família; Enfermagem pediátrica

\section{Keywords}

Family nursing; Professional-family relation; Pediatric nursing

\section{Descriptores}

Enfermería de la familia; Relaciones profesional-familia; Enfermería pediátrica

\section{Como citar:}

Marques FR, Mandetta MA. Intervenção educacional para promover o cuidado centrado na família em oncologia pediátrica: um estudo piloto. Rev Soc Bras Enferm Ped. 2021;21(2):141-9.

${ }^{1}$ Universidade Federal do Mato Grosso do Sul, Campo Grande, MS, Brasil.

${ }^{2}$ Escola Paulista de Enfermagem, Universidade Federal de São Paulo, São Paulo, SP, Brasil.

Conflitos de interesse: nada a declarar.

Submetido: 1 de Agosto de 2021 | Aceito: 10 de Dezembro de 2021

Autor correspondente: Fernanda Ribeiro Baptista Marques | E-mail: fer.rbmarques@gmail.com

DOI: http://dx.doi.org/10.31508/1676-379320210020 


\section{Introdução}

O Modelo do Cuidado Centrado no Paciente e na Família (MCCPF) é recomendado como uma das melhores práticas para promover a inclusão da família nos cuidados de saúde de seus membros e sua participação como membro da equipe de saúde. No entanto, na prática, sua implementação ainda está distante de se tornar realidade.

$\mathrm{Na}$ literatura, autores apontam as barreiras que interferem na promoção do cuidado com a família no hospital, como a falta de conhecimento e de preparo dos profissionais, a escassez de oferta de cursos de capacitação prática e de educação permanente sobre o cuidado com a família( ${ }^{(1)}$ e a falta de políticas institucionais que favoreçam e incentivem tais práticas, com a elaboração de protocolos e modelos que promovem o cuidado voltado para a família. ${ }^{(2)}$

Ademais, existem barreiras organizacionais que influenciam diretamente as ações de cuidado, como falta de tempo e de espaço para estabelecer as relações interpessoais, o que compromete o vínculo dos profissionais com a família, a comunicação e a negociação dos cuidados, instalações inadequadas nos ambientes assistenciais, além carga de trabalho pesada e o déficit de recursos humanos, que resultam em exaustão profissional. ${ }^{(2)}$

É preciso investir para minimizar essas barreiras e transformar o cuidado centrado no paciente e na família em uma realidade nas instituições de saúde. Para isso, devem-se capacitar os profissionais, para que consigam abordar a contento a família e incorporar novos conceitos sobre família ${ }^{(3)}$. Considera-se a sensibilização um dos meios para modificar a maneira de cuidar, perceber e atuar junto do paciente e da família. ${ }^{(4)}$

As intervenções educacionais têm sido o desafio atual dos pesquisadores na área de família e das instituições, uma vez que o processo de formação do profissional perpassa sua vivência, sua experiência e seu conhecimento prévio. ${ }^{(5,6)}$

A Enfermagem está intimamente ligada ao processo educativo, por essa razão, é necessário oferecer formação e oportunidade aos profissionais para que eles adquiram conhecimento e realizem uma prática fundamentada em preceitos teóricos e em evidências científicas. ${ }^{(6,7)}$

A educação permanente possibilita a construção de um conhecimento que promova modos de pensar inteligentes, criativos e profundos e tem a finalidade de favorecer o desenvolvimento pessoal e social, para que o indivíduo reflita sobre sua realidade. Isso o motiva a mudar, sob o ponto de vista pessoal e profissional, a partir do conhecimento adquirido, diminui as dificuldades que enfrenta em sua prática assistencial e estreita, cada vez mais, a lacuna entre teoria e prática. ${ }^{(8,9)}$

Acredita-se que o conhecimento deve ser promovido por meio de um processo de aprendizagem que esteja ancorado em um pressuposto pedagógico crítico-reflexivo, como a Teoria da Aprendizagem Significativa, aliado ao uso de metodologias inovadoras, que possibilitem uma aprendizagem significativa, a partir de reflexões sobre situações vivenciadas no dia a dia do trabalho. ${ }^{(10)}$ Assim, a introjeção de um novo conhecimento, por meio de pensamento reflexivo, dialógico, contextual, colaborativo e construtivo, adquire sentido para os profissionais e proporciona mudanças atitudinais no cuidado com o outro. ${ }^{(10)}$

Questiona-se, então, se a implementação de uma intervenção educacional fundamentada na Teoria da Aprendizagem Significativa possibilita a aquisição de novos conceitos sobre o MCCPF e modifica pensamentos, sentimentos e atitudes dos profissionais da área de Enfermagem em relação à família, no contexto da oncologia pediátrica.

O objetivo do estudo foi avaliar o impacto de uma intervenção educacional no conhecimento, na percepção e nas atitudes dos profissionais da equipe de Enfermagem em relação ao Modelo do Cuidado Centrado no Paciente e na Família.

\section{Métodos}

Estudo piloto do tipo quase-experimento com o delineamento de amostras temporais equivalentes em três fases: pré-intervenção, pós-intervenção imediata e três meses pós-intervenção.

O local de realização foi uma unidade de internação de um hospital especializado em oncologia pediátrica no município de São Paulo. Optou-se por uma unidade geral de internação com crianças em fase de diagnóstico, em tratamento quimioterápico, intercorrências clínicas, tratamento cirúrgico ou em fase final de vida, com 12 leitos para ser o piloto do projeto de implementação do MCCPF na instituição. 
Neste local as famílias são incentivadas a ficar com a criança, acompanhando-a nos exames diagnósticos, e nos tratamentos durante a hospitalização. A equipe de enfermagem que atua na unidade é composta por um enfermeiro coordenador, cinco enfermeiros assistenciais e 16 técnicos de enfermagem perfazendo um total de 22 profissionais divididos nos períodos matutino, vespertino e noturno.

A amostra foi constituída de profissionais da equipe de enfermagem de acordo com os critérios de inclusão: gestores e coordenadores da equipe de enfermagem, enfermeiros assistenciais e técnicos de enfermagem que atuavam na unidade de internação e enfermeiros residentes. Como critérios de exclusão: enfermeiros discentes dos cursos de Pós-graduação Lato Senso em Enfermagem e de Graduação, devido à sua rotatividade na unidade.

A pesquisa foi realizada no período de junho de 2015 a janeiro de 2016, seguiu todos os preceitos da Resolução 466/12 e foi aprovada pelo Comitê de Ética em Pesquisa da Instituição de Ensino Superior sob n ${ }^{\circ}$ : 1.044.363 e no: 1.388.161.

A intervenção educacional realizada foi um curso denominado 'Sensibilização para o Cuidado Centrado no Paciente e na Família', cujo marco teórico é fundamentado nos conceitos centrais do Modelo do Cuidado Centrado na Família (MCCF). ${ }^{(11)} \mathrm{O}$ eixo pedagógico adotado foi a Teoria da Aprendizagem Significativa, segundo Ausubel. ${ }^{(10)}$

O curso consistiu em três módulos e totalizou 12 horas, divididas em atividades presenciais e a distância. O módulo I, em que se abordou a filosofia do MCCPF, trazia conteúdo para a estrutura cognitiva do profissional e que explicavam sua relevância para a aprendizagem do novo material. No módulo II, debateu-se sobre as necessidades da criança com câncer em decorrência da doença e do tratamento e a transição do hospital para o domicílio. O módulo III trouxe considerações a respeito da prática profissional da equipe de Enfermagem com a família da criança com câncer, reforçou pontos já discutidos e introduziu, por meio do relato de uma narrativa de família, conceitos que precisam ser modificados e qualificados, deixando implícito, no depoimento do familiar, a diferença de atitudes dos profissionais diante da família.

Os recursos pedagógicos utilizados visaram facilitar o aprendizado, evitar a assimilação obliteradora e ativar o processo de aquisição de significados. Foram realizadas exposições teóricas dialogadas, discussão de caso clínico, análise de cenas de filmes e entrevista com uma família que contou sua experiência com manejo da doença e a transição do hospital para o domicílio. As aulas foram ministradas por convidados, especialistas no tema família, e pela própria pesquisadora.

Os dados foram coletados em três fases, denominadas de pré-intervenção - realizada entre os meses de junho e julho de 2015; pós-intervenção imediata - entre os meses de outubro e novembro de 2015 - e três meses pós-intervenção, realizada entre janeiro e fevereiro de 2016. Na fase de pré-intervenção, no início da primeira aula os membros da equipe foram convidados a participar do estudo e, depois que confirmaram o aceite, assinaram o Termo de Consentimento Livre e Esclarecido. Na sequência, cada participante preencheu um questionário com dados de caracterização sociodemográfica e foi aplicado um questionário de avaliação do conhecimento teórico sobre MCCPF, elaborado pela pesquisadora especialmente para este estudo. O questionário, composto de dez assertivas, requer respostas do tipo 'verdadeiro' ou 'falso'. Estabeleceu-se a pontuação de cada assertiva em 10 pontos, e a variação final entre zero e 100 pontos. Conforme o número de pontos obtidos, os profissionais foram categorizados nas seguintes classificações: desconhece (pontuação de zero a 20); conhece pouco (pontuação de 30 a 40); conhece razoavelmente (pontuação de 50 a 60); conhece bem (pontuação de 70 a 80 ) e conhece plenamente (pontuação de 90 a 100). Antes de ser aplicado, o instrumento passou por um processo de validação de conteúdo, com um painel de juízes. O índice de concordância obtido foi de $90 \%$ depois de duas rodadas, seguidas de validação semântica com profissionais da equipe de Enfermagem durante um evento, em que se avaliaram o tempo de preenchimento, a clareza, a pertinência e a adequação das questões. Os participantes dessa etapa não mencionaram dificuldades quanto à compreensão das questões, e o tempo de resposta variou entre três e cinco minutos. Assim, o instrumento foi considerado apto a ser utilizado no estudo.

Depois, aplicou-se o Percepção do Cuidado Centrado na Família - Equipe versão brasileira PCCF-E, composto de 20 questões, com perguntas claras e diretas sobre a percepção dos profissionais em relação ao $\mathrm{MCCF}$, que contemplaram os pressupostos 'respeito e 
dignidade', 'informação compartilhada', 'participação nos cuidados' e 'colaboração' As 20 questões são formadas de três domínios: respeito, colaboração e apoio. O domínio 'respeito' inclui seis itens, que reconhecem os direitos da família no hospital. O segundo, 'colaboração', envolve o reconhecimento do papel dos pais na parceria do cuidado do seu filho e compreende nove itens. O terceiro domínio - 'suporte' - inclui cinco itens, relativos à maneira como os profissionais da equipe de saúde oferecem suporte à família. As respostas são do tipo Likert, que variam em quatro opções: nunca, algumas vezes, geralmente e sempre, com pontuação de 1 a 4 , respectivamente. ${ }^{(12)}$ Cabe ressaltar que os valores atribuídos à questão 15 são empregados de forma inversa antes da análise por ser uma negativa. ${ }^{(12)}$

Por fim, aplicou-se a escala Importância das Famílias nos Cuidados de Enfermagem - Atitudes dos Enfermeiros IFCE-AE, desenvolvida na Suécia, traduzida e validada para a língua portuguesa de Portugal. Posteriormente, foi realizada equivalência semântica para a língua portuguesa brasileira. ${ }^{(13)}$ Trata-se de uma escala de autopreenchimento, composta de 26 itens com respostas do tipo Likert, que variam de 'discordo completamente', 'discordo', 'concordo' e 'concordo completamente'. O escore de cada item varia de 1 a 4, e o escore total, de 26 a 104. A escala é subdividida em três subescalas, que podem ser mensuradas como três dimensões independentes: (1) Família: Parceiro dialogante e recurso de coping, composta de 12 itens, cujo escore varia de 12 a 48; (2) Família: recurso nos cuidados de enfermagem, composta de 10 itens, cujo escore varia de 10 a 40; (3) Família: fardo, composta de quatro itens, cujo escore varia de 4 a 16. Cabe ressaltar que os valores atribuídos aos itens que compõem a subescala 3 são empregados de forma inversa antes da análise para obtenção do escore total. No entanto quando se quer analisar cada dimensão separadamente, não há inversão das repostas da dimensão três. Assim, interpreta-se que quanto maior o escore obtido nas duas primeiras dimensões e menor o escore obtido na terceira dimensão, mais importância os enfermeiros atribuem à família nos cuidados, ou seja, revelam mais atitudes de suporte. ${ }^{(13)}$

No final da última aula, cada participante respondeu os mesmos instrumentos de medida utilizados na pré-intervenção. Três meses depois da intervenção, os participantes foram convidados pessoalmente a responder os três instrumentos novamente.
Para compreender a participação dos profissionais no curso, realizou-se um estudo qualitativo utilizando-se a Análise Qualitativa de Conteúdo como referencial metodológico. ${ }^{(14)}$ A coleta dos dados do estudo qualitativo foi conduzida, depois de um convite para cada um dos que participaram das três fases da coleta dos dados quantitativos. As entrevistas semiestruturadas individuais foram realizadas por uma graduanda bolsista de um projeto de extensão, vinculada ao grupo de pesquisa, acompanhada pela orientadora deste estudo, a fim de evitar algum desconforto ou viés que interferisse nos resultados.

Para dar início à entrevista, utilizou-se a seguinte questão norteadora: Conte-me como é para você pensar, sentir e agir com a família depois de ter participado do curso. Outras perguntas também foram realizadas a fim de ampliar a compreensão sobre a experiência do profissional. As respostas dos participantes foram gravadas, com o consentimento deles.

Os dados quantitativos foram registrados em um banco de dados no programa Excel®, as variáveis categóricas, analisadas por meio de frequências absoluta (n) e relativa (\%), e as numéricas, por média, mediana, quartis e desvio-padrão.

Para analisar as variáveis relacionadas ao conhecimento, foi aplicado o teste $Q$ de Cochran, que compara a maior variância com as demais. Para analisar a percepção e a atitude dos profissionais, foi utilizado o teste de Friedman, que compara dados amostrais vinculados, ou seja, quando o mesmo indivíduo é avaliado mais de uma vez.

As associações entre as variáveis demográficas e o conhecimento foram analisadas por meio do Teste Exato de Fisher. Os testes Mann-Whitney e Kruskal-Wallis foram aplicados para analisar os domínios do PCCF-E versão brasileira e o escore total e as subescalas do instrumento IFCE-AE. O nível de significância adotado foi de $5 \%(p<0,05)$. Para as análises estatísticas, empregou-se o programa Statistical Package for the Social Sciences ${ }^{\circledR}$ (SPSS) para Windows ${ }^{\circledR}$, versão 19.

Para analisar os dados qualitativos, utilizou-se a análise indutiva, em que os dados partem do específico para o geral, e os casos particulares são observados e, em seguida, combinados em um todo. ${ }^{(14)}$ Assim, as transcrições dos discursos foram lidas na íntegra e extraídos códigos que foram analisados e agrupados em subcategorias, que receberam nomes provisórios de 
acordo com o significado. A seguir, foram constituídas as categorias inseridas nos temas, de acordo com suas similaridades e divergências. ${ }^{(14)}$

\section{Resultados}

A amostra empregada para a avaliação do impacto da intervenção educacional em relação ao conhecimento, às percepções e às atitudes dos profissionais de um hospital de oncologia pediátrica, foi composta de 18 profissionais da equipe de enfermagem, a maioria do sexo feminino - 88,9\% (16), com faixa etária entre 31 e 45 anos - 61,2\% (11), 66,6\% (12) técnicos de Enfermagem, $16,7 \%$ (3) enfermeiros assistenciais, e $16,7 \%$ (3) residentes de Enfermagem; e 61,2\% (11) com menos de um ano na instituição. Quanto ao contato prévio dos profissionais com o conteúdo sobre enfermagem da família, 89\% responderam afirmativamente. Desse total, $25 \%$ ocorreram durante a formação profissional na graduação; $25 \%$, em cursos de especialização, de extensão ou de capacitação na instituição; e 50\% durante o curso técnico. Isso demonstra que essa temática já vem sendo introduzida nos diversos cenários de ensino e de aperfeiçoamento profissional. Na figura 1 avalia o conhecimento teórico dos profissionais sobre o cuidado centrado no paciente e na família, nas fases de aplicação do questionário.

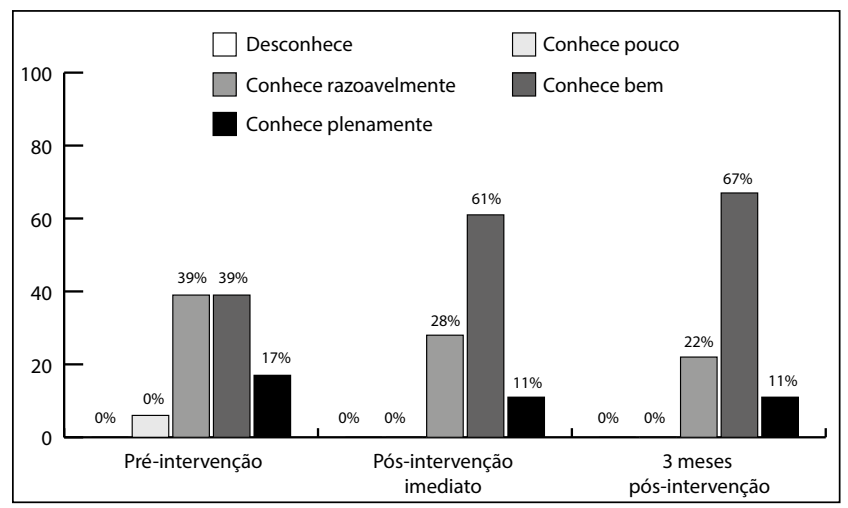

Figura 1. Conhecimento teórico dos profissionais da equipe de Enfermagem sobre o cuidado centrado no paciente e na família nas fases pré-intervenção, pós-intervenção imediata e três meses pós-intervenção $(n=18)$

Ao analisar cada questão separadamente, o padrão de respostas dos participantes não apresentou alteração significante nos períodos de tempo para a maioria das questões, com exceção da três (Os profissionais de saúde devem comunicar à família sobre escolhas realizadas pela equipe se saúde, relacionadas ao tratamento, ao plano terapêutico e à transferência do hospital para o domicílio.), em que houve erro de $28 \%$ na pré intervenção e redução do erro em $11 \%$ na pós-intervenção $(\mathrm{p}=0,050)$. Na questão cinco (Os pacientes e as famílias devem ser informados sobre os medicamentos - ações, efeitos colaterais e interações - e o tratamento para poderem participar ativamente do cuidado, negociando suas preferências?) houve acerto de $83 \%$ na pré-intervenção e de $100 \%$ na pós-intervenção $(p=0,050)$. Ao analisar cada domínio do instrumento PCCF-E versão brasileira (Figura 2), foi possível identificar um aumento dos valores das médias da dimensão 'Respeito' na fase de pré-intervenção $(3,22)$ para a fase 'três meses pós-intervenção' $(3,31)$; e da dimensão 'Suporte', que passou de 2,71 para 2,82, respectivamente. No que diz respeito à dimensão 'Colaboração', observou-se uma percepção mais positiva na fase de pré-intervenção $(3,15)$, diminuição, na fase de pós-intervenção imediata $(2,98)$, e elevação, na fase 'três meses pós-intervenção (Figura 2).

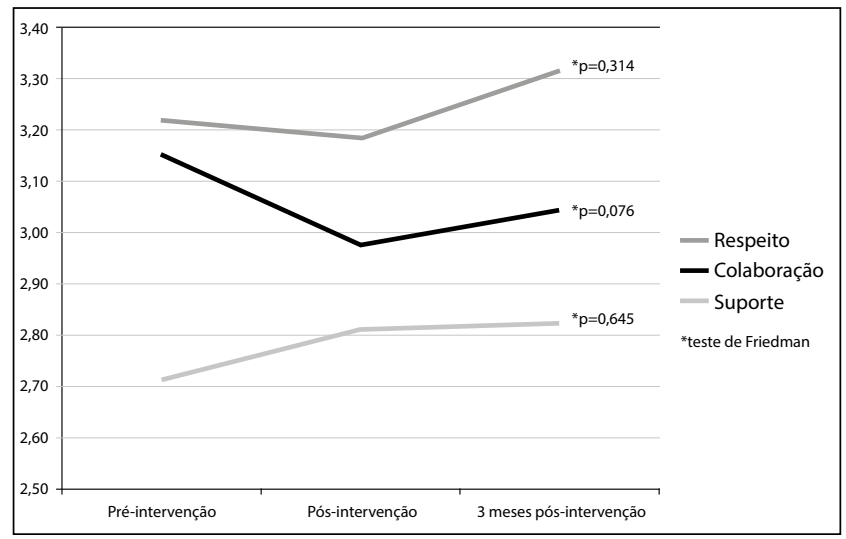

Figura 2. Resultados do teste de Friedman para escores médios das respostas dos membros da equipe de enfermagem por domínios no instrumento 'Percepção do cuidado centrado no paciente e na família - Equipe, versão brasileira $(n=18)$

Ressalta-se que, na Q4 - 'Os pais conseguem fazer perguntas sobre o tratamento de sua criança' - houve mudança significante $(p=0,020)$ nas respostas dos profissionais e uma percepção mais positiva da fase de pré-intervenção para a fase de pós-intervenção. $\mathrm{Na}$ Q15 - os pais (não) são sobrecarregados de informações que recebem sobre sua criança - constatou-se um movimento de mudança na percepção $(p=0,029)$ da 
pré-intervenção para as demais fases, o que indica que a intervenção educacional aliviou essa sobrecarga. Em relação à atitude dos profissionais da equipe de enfermagem sobre a importância da família nos cuidados de enfermagem, observou-se, pelos valores da moda, que, nas subescalas 1 - 'Família parceiro dialogante e recurso de coping' e 2 - 'Família: recurso nos cuidados de enfermagem', a opção "concordo" foi assinalada com mais frequência nas três fases de utilização do instrumento, enquanto na subescala 3 - 'Família: fardo', a opção "discordo" foi a mais assinalada em todas as fases. Na pré-intervenção, a opção 'concordo' foi assinalada em 21 itens do instrumento; na pós-intervenção imediata, em 23 itens; e na fase três meses pós-intervenção, em 25 itens. Isso revelou que os participantes apresentam uma atitude positiva em relação à família e se mantêm de maneira equilibrada nessa opção, com algumas flutuações. O estudo mostrou, ainda, um movimento de mudança de atitude dos profissionais em relação à família na fase de pré-intervenção para as demais fases do estudo, que está representado nos itens Q3 - Uma boa relação com os membros da família me dá satisfação no trabalho - $(\mathrm{p}=0,010) ; \mathrm{Q} 4$ - Os membros da família devem ser convidados a participar ativamente dos cuidados de enfermagem do paciente - $(\mathrm{p}=0,001)$; $\mathrm{Q} 6$ - No primeiro contato com os membros da família, convido-os a participar das discussões sobre o processo de cuidados do paciente - $(\mathrm{p}=0,018)$; Q18 - Considero os membros da família como parceiros - $(\mathrm{p}=0,013)$ e Q25 - Vejo-me como um recurso para as famílias, para que elas possam lidar o melhor possível com a sua situação - $(p=0,007)$. Quanto às demais questões, não houve alteração no padrão de respostas dos profissionais da equipe de enfermagem da fase de pré-intervenção para as demais.

A escala total IFCE-AE apresentou média igual a $75(\mathrm{DP} \pm 8,58)$, a qual variou de 63 (mínimo) para 102 (máximo) na fase de pré-intervenção; 78 (DP $\pm 7,48)$, na fase de pós-intervenção imediata, com variação de 67 (mínimo) e 96 (máximo); e 79 (DP $\pm 8,15)$, com variação de 67 (mínimo) e 97 (máximo) em três meses pós-intervenção. Esses valores evidenciam que os profissionais da equipe de enfermagem aumentaram a atribuição de importância da inclusão da família nos cuidados. Na fase de pré-intervenção, a metade dos participantes apresentou escore total médio de 73, que é próximo da média total da escala (75). Além disso,
$25 \%$ deles obtiveram escore total inferior a 65 (quartil 1), o que indica que um quarto dos profissionais da equipe de enfermagem teve, naquela ocasião, atitudes que atribuem pouca importância à inclusão da família nos cuidados de enfermagem. Mas, nas fases de pós-intervenção imediata e de três meses pós-intervenção, a metade dos participantes (quartil 2) mostrou escore total médio de 76, evidenciando um aumento nessa atribuição. No que se refere à subescala 1 - Família: parceiro dialogante e recurso de coping' - cujo escore pode variar entre 12 e 48, foi possível observar um aumento das médias da fase de pré-intervenção para as demais fases $(\mathrm{p}=0,003)$. Na pré-intervenção, o escore médio foi de $33(\mathrm{DP}=2,82)$, que variou entre 28 (mínimo) e 38 (máximo), sendo que a metade dos profissionais apresentou escore 33. Na fase de pós-intervenção imediata, o escore médio foi de 36 ( $\mathrm{DP}=3,34)$, com variação de 27 (mínimo) e 43 (máximo), e a metade dos profissionais obteve escore 36; já na fase de três meses pós-intervenção, o escore médio foi de 37 ( $\mathrm{DP}=3,60)$, com variação mínima de 32 e máxima de 44 . A metade dos profissionais apresentou escore 36 . Tais resultados revelam melhora na atribuição de importância à família. Na subescala 2 - Família: recurso nos cuidados de enfermagem', não foi possível observar alteração entre os valores das médias (31), conforme a Figura 3. O escore na pré-intervenção variou entre 24 (mínimo) e 38 (máximo); na pós-intervenção imediata, entre 23 (mínimo) e 39 (máximo); e 3 meses pós-intervenção, 23 (mínimo) e 41 (máximo). Metade dos participantes desta pesquisa apresentou escore aproximado de 30 na fase de pré-intervenção, que está muito próximo da média dos participantes em todas as fases. $\mathrm{Na}$ subescala 3 - Família: fardo - cujo escore pode variar entre 4 e 16, a média na pré-intervenção foi de 11, com um aumento na pós-intervenção imediata e três meses pós-intervenção, com média de 12 . O escore na pré-intervenção variou entre 9 (mínimo) e 14 (máximo); na pós-intervenção imediata, entre 9 e 16; e de três meses pós-intervenção, entre 9 e 15. Metade dos participantes obteve escore 11 na pré-intervenção, 12, na pós-intervenção imediata, e 11, de três meses depois da intervenção, cujos valores estão próximos das médias gerais e podem ser encontrados nos quartis 2 e 3 (Figura 3).

A análise qualitativa realizada possibilitou aprofundar a compreensão do impacto do curso para os 


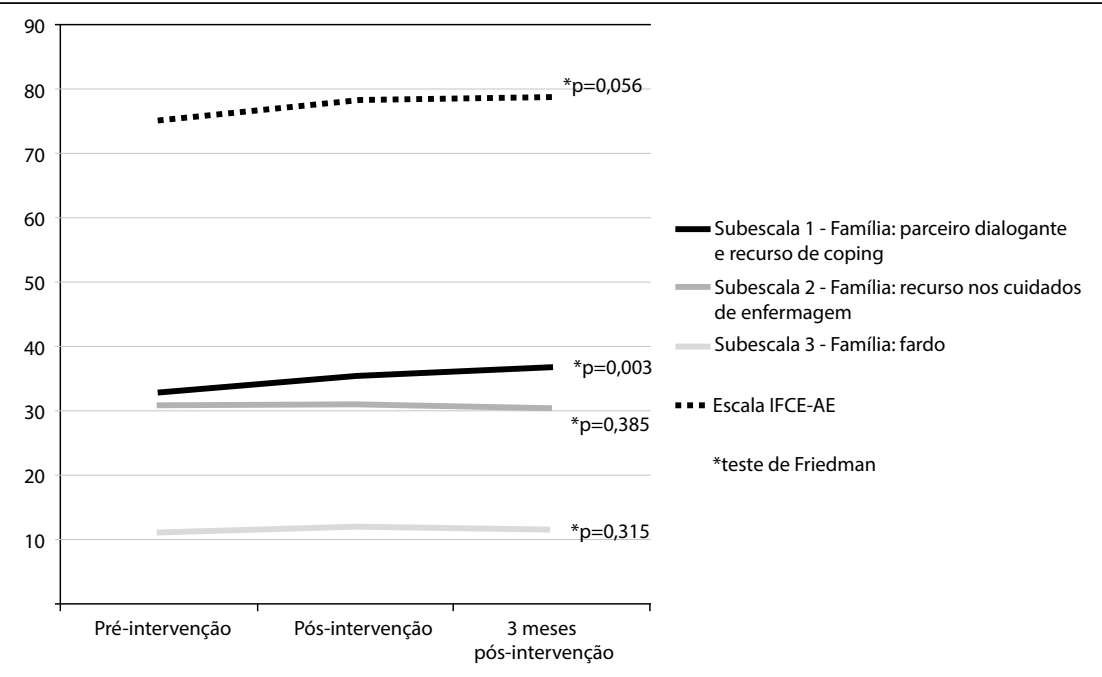

Figura 3. Resultados do teste de Friedman para os escores médios das respostas dos membros da equipe de enfermagem na escala 'Importância das Famílias nos Cuidados de Enfermagem - Atitudes dos enfermeiros' - e nas subescalas ( $n=18)$

participantes. O tema 'Tornar-se sensível à família' representa a aproximação dos profissionais com a temática, que se torna significativa à medida que lhes é oferecida uma oportunidade para se aproximarem da experiência de sofrimento da família com uma criança hospitalizada com câncer e dos pressupostos do MCCPF. A sensibilização dos participantes no curso foi intermediada pela metodologia de ensino aplicada, em que se utilizaram estratégias educacionais inovadoras em cada módulo, que foram fundamentais para remodelar o processo de cuidado inserido em cada participante. Assim, o significado central 'Tornar-se sensível à família' representa a mudança ocorrida nos profissionais identificada a partir da abstração das categorias 'Sentir-se compadecido com a experiência da família'; 'Pensar na família de maneira conceitual' e 'Ir ao encontro do cuidado da família'. Na categoria 'Sentir-se compadecido com a experiência da família', a oportunidade de ouvir a experiência família com doença e hospitalização durante o curso sensibiliza os profissionais de enfermagem. Percebem que a aproximação com a experiência de dor da família exemplificada em cenas de filmes e depoimentos de famílias que vivenciaram a hospitalização; assim como a experiência de transição para o domicílio, os torna mais sensíveis ao sofrimento da família e passam a sentir compaixão pela família, incluindo sentimentos de benevolência, empatia e tolerância. A categoria "pensar na família de modo conceitual "revela que o aprendizado sobre os pressupostos teóricos da abordagem do Cuidado Centrado na
Família amplia o pensamento dos profissionais sobre família e ressignifica o conceito de família, o que lhes permite se aproximar mais das famílias, sensibilizados pela abordagem de cuidado. Na categoria "Ir ao encontro do cuidado da família" profissionais sensibilizados passam a pensar sobre como encontrar maneiras para modificar sua prática. Quando compreendem os pressupostos do MCCF, eles sentem necessidade de ficar mais próximos da família e vão ao seu encontro para aplicar os conceitos aprendidos no curso ao seu cotidiano de trabalho, incluindo a família e negociando os cuidados com sua participação. Percebem que, quando conseguem isso, aproximam-se muito mais da família. No entanto, revelam que ainda há barreiras institucionais e pessoais que dificultam o seu agir em relação à família.

\section{Discussão}

A intervenção educacional favoreceu nos profissionais a aquisição de conhecimentos sobre o MCCPF que interagiram com os conceitos prévios existentes, advindos de cursos ou de sua experiência pessoal e profissional, e passaram a ter um novo significado, tornando-os sensibilizados para essa abordagem de cuidado. As estratégias de ensino aplicadas se revelaram capazes de favorecer a discussão e reflexão sobre o tema, revelando-se fundamentais para a sensibilização dos profissionais sobre o cuidado a família. 
O Instituto do Cuidado Centrado no Paciente e na Família recomenda que os pacientes e as famílias participem da elaboração de programas de ensino; que sejam aplicadas estratégias inovadoras, como simulação, jogos interativos e outros recursos da mídia digital na graduação, na pós-graduação e em programas de educação permanente, para fomentar uma prática interprofissional colaborativa sustentada pelo MCCF.(15)

O uso da narrativa da família para sensibilizar os profissionais tem sido preconizado, pois provoca uma aproximação com a experiência da família e faz com que eles percebam o impacto da doença, as dificuldades enfrentadas e a força da família para superar as adversidades. A narrativa promove a assimilação da teoria com a prática e motiva os profissionais a buscarem mais elementos para seu aprendizado. Além disso, quando ouvem uma narrativa, refletem sobre a prática com a família e passam a repensar seu agir, o que lhes provoca uma disposição interna para pensar na família como foco do cuidado. ${ }^{(16)}$

Um grupo de pesquisadoras portuguesas elaborou um jogo denominado de Family Nursing Game, com o intuito de ensinar os profissionais sobre o conteúdo de família. Essa estratégia foi válida para incorporar novas concepções de família e uma poderosa ferramenta para motivar, potencializar a aprendizagem, estimular a interação do grupo, fomentar a reflexão e proporcionar momentos ricos de trocas de experiências. ${ }^{(17)}$

Estudos realizados no cenário nacional e no internacional revelaram a importância das intervenções educativas para promover o cuidado com a família. ${ }^{(18-20)}$ Um estudo brasileiro, em que se mediram as percepções dos profissionais em relação ao $\mathrm{MCCF}$ e ao estresse parental em uma unidade neonatal, depois da implementação do MCCF, constatou que os profissionais se mostravam mais positivos quanto à participação da família ampliada nos cuidados, à permanência dos pais com a criança durante procedimentos, à inclusão da família no cuidado da criança e ao reconhecimento da rede de suporte dos pais. Os autores registraram uma melhora estatisticamente significativa na percepção dos profissionais da equipe de saúde na fase de pós-intervenção, em relação ao acolhimento da família. ${ }^{(18)}$

Na Suécia, verificou-se que enfermeiros apresentaram mais atitudes de apoio ao envolvimento das famílias nos cuidados de enfermagem. O escore médio geral da escala Families' Importance in $\mathrm{Nu}$ rsing Care-Nurses' Attitudes (FINC-NA) foi de 88, ${ }^{(19)}$ assim como enfermeiros de unidades de pediatria e materno-infantil de um hospital universitário brasileiro, cujo escore médio geral da escala FINC-NA foi de $82 .{ }^{(20)}$

Neste estudo, os profissionais não apresentaram mudança nos escores na subescala 3 - Família: Fardo, nos três tempos. A concepção de que a família é um fardo está relacionada à ideia de que o enfermeiro não dispõe de tempo para cuidar das famílias e que elas são indesejáveis nos ambientes de cuidado em saúde. ${ }^{(20)}$ Considerar a família um fardo é um obstáculo para que se estabeleça um relacionamento colaborativo entre os enfermeiros e as famílias e, como neste estudo, está atrelado às barreiras pessoais, organizacionais e ambientais. ${ }^{(20)}$

De acordo com os resultados, os participantes foram, aos poucos, sensibilizados pelo novo modo de "pensar na família". Por causa disso, reviram suas crenças e valores, iniciaram um movimento de mudança de atitude com a família, aproximaram-se mais de sua experiência, passaram a ouvir suas demandas e promoveram sua inclusão nos cuidados.

Entende-se que, para mudar um local, é necessário iniciar uma mudança pessoal. Para isso, é indispensável a parceria entre o educador e o aprendiz. Sensibilizar é uma estratégia que possibilita uma aprendizagem significativa, mas precisa de avaliação e de continuidade. Ademais, as reflexões realizadas tornaram os profissionais mais críticos em relação à sua prática e lhes possibilitou identificar as barreiras institucionais e pessoais que comprometem a adoção desse modelo de cuidado, como a falta de política sobre Modelo do Cuidado Centrado no Paciente e na Família, comprometimento do dimensionamento do pessoal nas unidades, sobrecarga de trabalho e escassez de tempo, assim como a falta de preparo para lidarem com as demandas da família.

Como limitação, aponta-se a realização do estudo com um pequeno número de profissionais da equipe de Enfermagem, em uma unidade de internação, sem a inclusão de outros profissionais da equipe multidisciplinar de saúde. Recomenda-se que essa intervenção educacional seja aplicada com todos os profissionais que atuam na instituição. 


\section{Conclusão}

Em relação ao Conhecimento Teórico, houve a assimilação de novos conceitos, sendo significante, em relação à importância da oferta de informações à família, ao seu envolvimento nos cuidados e à negociação de suas preferências com a equipe.

Quanto a Percepção do Modelo do Cuidado Centrado no Paciente e Família houve mudança com a intervenção, no domínio Respeito, em relação à maior abertura para os pais fazerem perguntas e, no domínio Colaboração, com menor sobrecarga de informações aos pais sobre o tratamento da criança.

Em relação à Atitude dos profissionais com a família, houve mudança significante, representada pela satisfação no trabalho a partir de uma boa relação com a família; pelo convite à família para participar ativamente dos cuidados e discussões, considerando-a parceira do cuidado e o profissional como um recurso para as famílias lidarem melhor com a situação.

O estudo qualitativo ampliou a compreensão do processo de aprendizagem dos profissionais da equipe de enfermagem em relação à intervenção educacional, complementando os achados da pesquisa quantitativa. Na perspectiva dos profissionais de saúde, a intervenção educacional favoreceu a aquisição de novos conhecimentos teóricos para a prática, ajudou-os a ampliar seu pensamento sobre família e modificou seu conceito e suas atitudes com a família.

Para tornar o MCCPF uma realidade nos serviços de saúde é preciso que seus princípios sejam incorporados à filosofia institucional, norteando a cultura organizacional. Ademais é preciso haver um Programa de Educação Permanente incluindo todos os profissionais de saúde, a fim de fomentar o desenvolvimento de habilidades de comunicação, de negociação e de mediação de conflitos, fundamental para o envolvimento da família nos cuidados, visando à satisfação do usuário e do profissional.

\section{Agradecimentos}

O presente trabalho foi realizado com apoio da Coordenação de Aperfeiçoamento de Pessoal de Nível Superior - Brasil (CAPES).

\section{Colaborações}

Marques FR e Mandetta MA contribuíram com a concepção do projeto, análise e interpretação dos dados, redação do artigo, revisão crítica relevante do conteúdo intelectual e aprovação da versão final a ser publicada.

\section{Referências}

1. Sellán AV, Soto MC, Martinez ML. Family-centered care: a philosophy to be developed. Pediatric Neonatal Nurs Open J. 2017;5(1):1-5.

2. Coats $H$, Bourget E, Stark H, Lindhorst T, Saiki-Craighill S, Cutis J R, et al. Nurses's reflections on bebefits and challenges of implementing family- centered care units. Am J Crit Care. 2018;27(1):52-8.

3. Parent K, Jones K, Phillips L, Stojan JN, House JB. Teaching patient- and familycentered care: integrating Shared Humanity into Medical Education Curricula. AMA J Ethics. 2016;18(1):24-32.

4. Lown BA, McIntosh S, Gaines ME, McGuinn K, Hatem DS. Integrating Compassionate, Collaborative Care (the "Triple C") Into Health Professional Education to Advance the Triple Aim of Health Care. Acad Med. 2016;91(3):310-6.

5. Leko MM, Roberts C, Peyton D, Pua D. Selecting Evidence-Based Practices: What Works for Me. Interv Sch Clin. 2019;54(5):286-94.

6. Horntvedt MT, Nordsteien A, Fermann T, Severinsson E. Strategies for teaching evidencebased practice in nursing education: a thematic literature review. BMC Med Educ. 2018;18:172.

7. Barton G, Bruce A, Schreiber R. Teaching nurses teamwork: Integrative review of competencybased team training in nursing education. Nurse Educ Pract. 2018;32:129-37.

8. Amaro MO, Mendonça ET, Carvalho CA, Nakada KN, Siman AG, Ferreira NC. Nurse concepts and practices on continuous education in hospital environment. Arq. Cienc Saúde. 2018;22( 2):87-94.

9. Abdullah, Zeb A, Ullah H, Bano N. Effectiveness of active learning in Nursing Education. Int J Sci Res Publ. 2017;7(6):588-90.

10. Ausubel DP. Aquisição e retenção de conhecimentos: uma perspectiva cognitiva. 1a ed. Plátano Editora; 2003. 219p.

11. Institute for Patient- and Family-Centered Care. What is patient- and familycentered care? [cited 2021 Dec 10]. Available from: http://www.ipfcc.org/about/pfcc.html

12. Silva TO, Alves LB, Balieiro MM, Mandetta MA, Tanner A, Shields L. Cross-cultural adaptation of an instrument to measure the family-centered care. Acta Paul Enferm. 2015;28(2):10712.

13. Figueiredo S, Margarida M, Ferreira SR, Martinho MJ, Figueiredo CA. Attitudes of nurses towards families: validation of the scale families' importance in nursing care - nurses atitudes. Rev Esc Enferm USP. 2011;45(6):1331-7.

14. Mills J. Qualitative methodology pratical guide. 1a ed. SAGE; 2014. 288p.

15. Institute for Family-Centered Care. Partnering with patients and families to design a patientand family-centered health care system: recommendations and promising practices [Internet]. [cited 2020 Mar 10]. Available from: http://www.ipfcc.org/faq.html

16. Amador DD, Marques FR, Duarte AM, Balbino FS, Balieiro MM, Mandetta MA. Use of narrative as an awareness strategy for a Family-Centered Care model. Rev Gaúcha Enferm. 2015;36(1):98-103.

17. Fernandes CS, Martins MM, Gonçalves LH. Another way to teach family: family nursing game. Acta Sci Heal Sci. 2014;36(2):195-200.

18. Balbino FS, Balieiro MM, Mandetta MA. Measurement of family-centered care perception and parental stress in a neonatal unit. Rev Latino-Am Enfermagem. 2016;24:e2753.

19. Svavarsdottir EK, Sigurdardottir AO, Konradsdottir E, Stefansdottir A, Sveinbjarnardottir EK, Ketilsdottir A, et al. The process of translating family nursing knowledge into clinical practice. J Nurs Scholarsh. 2015;47(1):5-15.

20. Cruz AC, Angelo M. Impact on nurses' attitudes of an educational intervention about Family Systems Nursing. Rev Enferm. 2018;26:e34451. 